Cahiers d'études africaines

$179-180 \mid 2005$

Esclavage moderne ou modernité de l'esclavage?

\title{
Sartre postcolonial ? Relire Orphée noir plus d'un demi-siècle après
}

\section{Kathleen Gyssels}

\section{OpenEdition}

1 Journals

Édition électronique

URL : http://journals.openedition.org/etudesafricaines/14952

DOI : 10.4000/etudesafricaines. 14952

ISSN : $1777-5353$

Éditeur

Éditions de l'EHESS

\section{Édition imprimée}

Date de publication : 19 décembre 2005

Pagination : 631-650

ISBN : 978-2-7132-2049-4

ISSN : 0008-0055

Référence électronique

Kathleen Gyssels, "Sartre postcolonial ? Relire Orphée noir plus d'un demi-siècle après », Cahiers d'études africaines [En ligne], 179-180 | 2005, mis en ligne le 01 janvier 2007, consulté le 16 juin 2020. URL : http://journals.openedition.org/etudesafricaines/14952 ; DOI : https://doi.org/10.4000/ etudesafricaines.14952 


\title{
CA I R N
}

chercher : repérer : avancer

Cet article est disponible en ligne à l'adresse :

http://www.cairn.info/article.php?ID_REVUE=CEA\&ID_NUMPUBLIE=CEA 179\&ID_ARTICLE=CEA 1790631

\section{Sartre postcolonial ? Relire Orphée noir plus d'un demi-siècle après}

\author{
par Kathleen GYSSELS
}

\section{Editions de l'EHESS | Cahiers d'études africaines}

2005/3-4 - 179

ISSN 0008-0055 | ISBN 2713220491 | pages 631 à 650

Pour citer cet article :

- Gyssels K., Sartre postcolonial ? Relire Orphée noir plus d'un demi-siècle après, Cahiers d'études africaines 2005/34, 179, p. 631-650.

Distribution électronique Cairn pour Editions del’EHESS.

(C) Editions de l'EHESS. Tous droits réservés pour tous pays.

La reproduction ou représentation de cet article, notamment par photocopie, n'est autorisée que dans les limites des conditions générales d'utilisation du site ou, le cas échéant, des conditions générales de la licence souscrite par votre établissement. Toute autre reproduction ou représentation, en tout ou partie, sous quelque forme et de quelque manière que ce soit, est interdite sauf accord préalable et écrit de l'éditeur, en dehors des cas prévus par la législation en vigueur en France. Il est précisé que son stockage dans une base de données est également interdit. 
À l'occasion du centenaire de la naissance de Jean-Paul Sartre, nous publions ici I'article de Kathleen Gyssels consacré aux rapports qu'entretient l'auteur de La Nausée avec le postcolonialisme. Peuton considérer celui-ci, notamment à travers sa longue introduction à I'Anthologie de la nouvelle poésie nègre et malgache de langue française de Léopold Sédar Senghor ou sa préface aux Damnés de la terre de Frantz Fanon, comme un précurseur ou un inspirateur de ce courant de pensée? Peut-on, par ailleurs, analyser ses positions tiers-mondistes - et les limites de celles-ci — à la lumière du postcolonialisme? Telles sont les questions que pose cet article et auxquelles il tente de répondre.

Jean-Loup AMSELLE

\section{Kathleen Gyssels}

\section{Sartre postcolonial ? Relire Orphée noir plus d'un demi-siècle après}

Sartre, tiers-mondiste et anticolonialiste

Outre ses nombreux articles de presse, essais et conférences rassemblés dans Situations $V$, et disponibles aujourd'hui en anglais sous le titre Colonialism and Neocolonialism (2001), Jean-Paul Sartre est le préfacier de trois essais cruciaux pour le développement des études postcoloniales : une préface à la première anthologie de poètes noirs, éditée par le poète sénégalais L. S. Senghor, en 1948, Orphée noir ; une à l'essai du Martiniquais Frantz Fanon, Les Damnés de la terre, publiée en 1956 ; et une troisième à l'essai du juif tunisien Albert Memmi, Portrait du colonisé, éditée en 1957.

À trois reprises, Sartre occupe donc le rôle d'instigateur et de promoteur comme le furent les mécènes blancs pour les premiers récits littéraires des Africains-Américains, les slave narratives improprement appelés «autobiographies des ex-esclaves » en français (Gyssels 2004). Cette inféodation d'auteurs noirs mérite d'être soulignée, tant il est vrai qu'André Breton fit 
de même pour Aimé Césaire lorsqu'il lança son Cahier d'un retour au pays natal (1939) et Robert Desnos pour Léon-Gontran Damas pour Pigments (1937), recueil illustré de surcroît par la reproduction d'une gravure du pacifiste flamand, Frans Masereel.

Avec ces noms, Senghor, Sartre, Damas et Césaire ${ }^{1}$, un réseau d'avantgarde gauchiste s'ouvre, un groupe très soudé de poètes et d'écrivains ${ }^{2}$ - souvent aussi politiciens — , qui ont considéré, en phase avec les surréalistes et les jazzmen ${ }^{3}$, leur plume comme «l'arme miraculeuse » pour changer le monde, permettre que le Noir, où qu'il soit, puisse «faire peau neuve », se décoloniser, se désaliéner. Tous ces intellectuels se répondent sans toutefois toujours se correspondre, tous écrivent les uns aux autres.

À l'occasion du centenaire de la naissance de Jean-Paul Sartre, j'aimerais relire la toute première préface qu'il a rédigée et qui lui valut déjà le surnom de philosophe «tiers-mondiste». Rédigé avec les meilleures intentions, ce «paratexte » reste un chapitre critique délaissé des Situations $I V$ comme par la plupart des sartriens. Est-ce à cause de sa longueur (44 pages ${ }^{4}$ ) ou d'une « appropriation de voix » qui ne lui est pas facilement accordée ?

En 1948, Sartre introduit la première anthologie de littérature « nègre », réunissant des auteurs guyanais, martiniquais, guadeloupéens, haïtiens, d'Afrique noire, d'une part, et «malgaches $»^{5}$, de l'autre. Dès le titre, la nouveauté est radicalement soulignée: Anthologie de la nouvelle poésie nègre et malgache de langue française (1948), ce qui est curieux puisque l'on peut être sûr qu'à l'époque le lecteur français n'avait aucune connaissance de l' « ancienne » poésie «nègre » et « malgache ». Dans l'après-guerre, on ne connaissait tout simplement pas la littérature «nègre et malgache » et « nouvelle » s'entend ici en fait au sens de «première », « neuve », n'ayant pas d'antécédents, du moins, d'expression française ${ }^{6}$. À moins que l'adjectif « nouvelle » renvoie à une autre anthologie complètement passée inaperçue,

1. Qui occupera la même fonction de mentor pour d'autres « débutants ». Je pense au Guyanais Bertène Juminer pour Les Bâtards, Présence africaine, 1961.

2. Il faudrait y ajouter Claude Lévi-Strauss et Michel Leiris, spécialiste des Antilles après sa mission de l'UNESCO: Contacts de civilisation en Martinique et en Guadeloupe (LeIRIS 1955) reste un essai fondamental pour tout chercheur dans ce domaine.

3. Pour lesquels Sartre se passionna également. Voir à ce propos GoRdon (2005).

4. En chiffres romains, que nous indiquons, ici, par des chiffres arabes pour la commodité de la lecture.

5. L'ordre tel qu'agencé par Senghor commence et finit par les deux territoires insulaires continentaux, la Guyane d'une part, et Madagascar de l'autre. Quant à «malgache », l'adjectif désigne les auteurs des Mascareignes, l'ensemble des îles françaises appelées comme «la Polynésie française»: La Réunion et Maurice et la grande île continentale, Madagascar.

6. Je renvoie ici à la très riche tradition des hain-tenys malgaches, en langue indigène et aux épopées bambara et wolof, pareillement en idiomes africains. Étrangement, à l'intérieur de l'Anthologie, le lecteur pourra goûter à quelques pages créolophones, plusieurs poèmes du Martiniquais Gilbert Gratiant étant ici présentés dans leur forme bilingue : créole sur la page de gauche, français sur la page de droite. 
celle de Léon-Gontran Damas (1947) parue un an avant, sous un titre bien neutre et sec, effaçant toute origine ethnique ou géographique, Poètes d'expression française ${ }^{7}$. Permettez-moi de rappeler combien 1'Introduction de Senghor devient alors fortement ironique, puisque celui-ci prend soin de signaler que l'Anthologie voit le jour à une occasion hautement mémorable, celle de la fête du «centenaire de la Révolution de 1848 »(Senghor, p. 1). Quelle servilité déployée ici, car 1848 est, pour les poètes antillais, une date capitale, celle de l'abolition définitive de l'esclavage aux Antilles, à la Réunion et en Guyane française ! De surcroît, quel « oubli » suspect de la part de l'académicien Senghor de ne pas signaler l'autre œuvre qui a devancé d'une année, sans préface, cette commémoration littéraire qui doit, une fois de plus, célébrer la République française et non rappeler aux peuples rendus amnésiques, malades «d'indigestion de tout morceau d'histoire de France » («Rappel », poème cité dans l'Anthologie, p. 10) ses déboires et ses massacres, sa déshumanisation et son commerce transatlantique.

Bref, l'adjectif devant la « poésie » demeure énigmatique, tout en ayant l'effet d'attirer le regard des lecteurs potentiels et de raviver, qui sait, leur faim. Outre quelques exceptions — et cela n'a pas essentiellement changé les lecteurs français ignoraient de facto les auteurs des Colonies et des exColonies françaises, tant il est vrai qu'en 1948 aucune ex-Colonie française (à part Haïti) n'avait acquis son indépendance.

L'Anthologie n'en devient que plus symbolique car elle opère un double but, à la fois émancipateur et, malgré elle, néo-colonialiste. Non seulement il s'agit de promouvoir une toute nouvelle littérature, une poésie jamais vue, parce qu'œuvre d'Africains, d'Antillais et de Malgaches, mais aussi d'introduire tout doucement une « francophonie », une littérature non hexagonale, une littérature d'Outre-mer. Ce qui rassemble ces poètes d'horizons quand même très divers (Madagascar n'a pas connu l' " univers de plantation » comme la Martinique, par exemple), de cultures et de régions fort éloignées les unes des autres (Césaire ne pourra s'aligner sur la poésie d'épigone d'un Birago Diop), c'est le fait qu'ils se servent du français comme langue littéraire. Colonisés par la France, ils ont en commun d'avoir été, ou pire, d'être toujours (puisque les Antilles ont voté, sous l'égide d'Aimé Césaire, la départementalisation en 1946) des «sujets de la France », de la République qui se pense métissée, mais qui ne l'est pas dans le quotidien (Dubois 2000). Sujets francophones d'ères coloniales disparates, ces auteurs auront donc assimilé la langue de l'ex-colonisateur au point d'en faire l'instrument d'émancipation juridique et politique. La nouvelle poésie instaure dès lors un champ qui va devenir très important, voire crucial, pour le rayonnement de la littérature française : la «francophonie » voit le jour grâce au même Senghor, aux présidents marocain Bourguiba et français de Gaulle, en 1962. D'où une certaine ambivalence de cette préface qui prétend émanciper pour mieux atteler, comme un appendice "exotique », des auteurs d'(ex-)Colonies françaises. D'où aussi les nombreuses contradictions dans

7. Daniel Maximin m'annonce une réédition sous son égide chez Présence africaine. 
ce discours préfaciel d'un des plus grands auteurs et philosophes marxistes que compta la République des lettres ${ }^{8}$. («Cette poésie », répétera Sartre tout le temps, «n'est pas pour des Blancs, mais cependant il la lira et nous la lirons »; «Cette poésie », insistera Sartre, est une poésie « révolutionnaire », mais en même temps, elle est tout entière marquée par le silence, vu les tabous et les inédits, l'indicible d'une condition coloniale et l'intraduisible de l'asservissement séculaire, «blessure » inguérissable du peuple colonisé dont le poète se veut le porte-parole $)^{9}$.

Le philosophe existentialiste accepte humblement ${ }^{10}$ de préfacer une chrestomathie composée par son ami et confrère, le Sénégalais L. S. Senghor, co-fondateur avec le Martiniquais Aimé Césaire et le Guyanais Léon-Gontran Damas (Gyssels 2002) d'une première «école littéraire » noire qui s'autobaptise négritude, néologisme greffé sur l'injurieux «nègre ». Que le génie nègre soit digne d'intérêt, le titre mythologique le souligne d'emblée: Orphée noir associe la négritude à la poésie des Antiques; le préfacier introduit par ailleurs de doctes comparaisons du genre : «Par une générosité suprême, [les poètes de la négritude] abandonnent [leur orgueil], comme Philoctète abandonnait à Néoptolème son arc et ses flèches » (p. 42). Par

8. Considérant plusieurs « degrés » de négritude, le préfacier oscille entre plusieurs poésies et plusieurs styles - «les mots de Léro s'organisent mollement, en décompression, par relâchement des liens logiques, autour de thèmes larges et vagues » (p. 27), alors que ceux de Césaire sont «pressés les uns contre les autres et cimentés par sa furieuse passion» (p. 27) - les uns plus revendicateurs et résistants, révolutionnaires que les autres.

Il attribue à certains le trait «Poésie "percussion" ", pendant qu'il parle pour d'autres de «poésie inspirée par la tradition séculaire des griots ». Ailleurs, il lui reconnaît davantage un caractère "crispé », ce qu'il explique par le fait que certains poètes s'inspirent du folklore, tout en voulant dire/signifier autre chose.

9. Enfin, la poésie est traversée tout entière par le silence, et Sartre de citer Mallarmé : «Évoquer dans une ombre exprès, l'objet tu par des mots allusifs, jamais directs, se réduisant à du silence égal» (p. 20), et de renvoyer à «L'holocauste des mots » de Bataille.

Le préfacier a raison de mettre en relief les non-dits, les tabous, l'indicible. Dans une langue empruntée au colonisateur, le colonisé, rusé, va, lui, contredire, contester le Blanc. Sartre a compris que le poète noir court-circuite le langage : que « derrière la chute enflammée des mots, nous entrevoyons une grande idole noire et muette » (p. 21) qu'il renversera la hiérarchie. Et pour mieux convaincre le lecteur présumé européen, il donne l'exemple de l'inversion «noirceur de l'innocence » (au lieu de la «blancheur de l'innocence »), ou encore «les ténèbres de la vertu» (au lieu des lumières de la vertu), pour bien nous faire comprendre ce qui se produit dans la tête d'un Noir, dans l'esprit des poètes nègres d'entendre toujours la noirceur ou leur négritude associée à tout ce qui est le plus obscur, laid, répugnant, etc.

10. C'est seulement à la page 29 que Sartre capte la bienveillance de son audience en reconnaissant humblement, ou en feignant de reconnaître, qu'il n'est pas bien positionné pour nous faire connaître ceux qu'il appelle les évangélistes noirs, et cela pour deux raisons : d'abord, parce qu'il n'est pas nègre (ce qui confirme la vision essentialiste : la négritude est bien quelque chose d'impartageable avec le Blanc) ; ensuite, parce que le français n'aurait pas de mots pour décrire la négritude (d'où d'ailleurs son caractère « crispé »). 
la même image orphique, le critique qu'est Sartre camoufle sa difficulté à cerner cette poésie révolutionnaire. De fait, le renvoi au mythe d'Orphée qui, de son chant divin, séduisait Eurydice laquelle disparaît dès qu'Orphée se retourne pour l'apercevoir, fait de la négritude, malgré tout son effort interprétatif, un objet évanescent. Temps faible d'un mouvement dialectique, étape brève et transitoire vers une société sans races et sans distinction d'épiderme, ce premier mouvement littéraire est un anti-racisme blanc, selon Sartre, qui résultera vite en une littérature où le critère racial n'aura plus de «sens ».

Bien que Sartre soit «une bonne conscience », comme le précise en insistant l'auteur guadeloupéen Daniel Maximin ${ }^{11}$, et qu'il ait lu et relu les poèmes (surtout ceux des Antillais), cette poésie lui échappe au moment même où il pense bien la présenter au public métropolitain. Après un demisiècle, je relirai cette préface en signalant des aspects de style, des éléments de son argumentation, pour surtout m'arrêter à son interprétation. Enfin, il convient de s'interroger sur l'héritage sartrien pour les théoriciens postcoloniaux.

\section{Rage/race}

À relire la préface de Sartre, ce qui frappe d'abord c'est que l'auteur, par le biais d'une métalepse puissante, s'adresse directement aux lecteurs de l'Anthologie :

«Qu'est-ce donc que vous espériez, quand vous ôtiez le bâillon qui fermait ces bouches noires? Qu'elles allaient entonner vos louanges ? Ces têtes que nos pères avaient courbées jusqu'à terre par la force, pensiez-vous, quand elles se relèveraient, lire l'adoration dans leurs yeux ?» (p. 9) ${ }^{12}$.

Dans l'incipit, Sartre s'en prend directement aux fils de colons pour dénoncer une filiation de maîtres et de colonisateurs qui n'en finissent pas d'exploiter et de perpétuer le système colonial. La forme dialogique de l'incipit («vous ») oblige le lecteur à se sentir concerné par l'énoncé sartrien qui emprunte une image métaphorique capitale : celle que la sociologue et linguiste Dany Bébel-Gisler (1976) appelle « la parole jugulée ». Vu l'interdit de la parole et la défense de contester le colonisateur, de résister aux brimades et aux violences verbales et physiques, psychiques et autres du colon, la forme créative libre qu'est la poésie a mis longtemps à émerger. Par l'accumulation de questions, interrompues par une temporelle qui a valeur de conditionnelle, le préfacier inculque durablement l'image d'une révolte légitime, d'une violence justifiée qui va trouver sa traduction dans

11. Dans un article manuscrit, "Sartre et le Tiers(-Monde) » qu'il m'a gentiment fait lire et qui a été exposé à la Bibliothèque nationale de France.

12. Toutes les références renvoient à L. S. SENGHOR (1948). 
une littérature de refus, une poésie qui fait de l'injure «nègre », un postulat identitaire et un titre fièrement revendiqué. Le « Nègre authentique » pour Sartre est celui qui brandit sa négritude comme une lance, qui va cracher sur le Blanc:

«Un juif, blanc parmi les blancs, peut nier qu'il soit juif, se déclarer un homme parmi les hommes. Le nègre ne peut nier qu'il soit nègre ni réclamer pour lui cette abstraite humanité incolore : il est noir. Ainsi est-il acculé à l'authenticité : insulté, asservi, il se redresse, il ramasse le mot "nègre" qu' on lui a jeté comme une pierre, il se revendique comme noir, en face du blanc, dans la fierté » (p. 14).

Le style péremptoire, voire agressif, est un premier trait qui trouve tout son effet lorsqu'il est accompagné d'un raisonnement qui consiste à inverser systématiquement les places et les fonctions, les rôles et les stéréotypes attribués au Noir et au Blanc. Sartre met le colonisé à la place du colonisateur, le Noir à la place du Blanc, pour mieux convaincre son lecteur du renversement dialectique, de l'avènement postcolonial : en vrai hégélien, le maître est à présent renversé par l'(ex)-esclave. Sartre avertit de la fin d'une ère, celle des empires coloniaux, celle de la supériorité blanche, de l'arrogance européenne, de la prétention française. Mais, je le répète, ce lecteur qu'il vise, et que vise l'Anthologie, ne serait pas le Français. La poésie ne parle pas pour ou à lui :

«Tous ceux, colons et complices, qui ouvriront ce livre, croiront lire, par dessus une épaule, des lettres qui ne leur sont pas destinées. C'est aux noirs que ces noirs s'adressent et c'est pour leur parler des noirs; leur poésie n'est ni satirique ni imprécatoire : c'est une prise de conscience » (p. 11).

Ce sera un premier paradoxe du texte introductif: Sartre soutient que cette poésie est destinée aux Antillais, Africains et Malgaches, mais en même temps, il se contredit, car Damas, Césaire et Senghor s'adressent bien à des allocutaires « universels ».

Deuxième trait stylistique, à l'image des poètes qu'il étudie, Sartre se fait contestataire, comme si sa bouche enfin s'était délivrée d'un mors :

«Il n'est pas vrai que le noir s'exprime dans une langue étrangère, puisqu'on lui enseigne le français dès son plus jeune âge et puisqu'il y est parfaitement à son aise dès qu'il pense en technicien, en savant ou en politique. Il faudrait plutôt parler de décalage léger et constant qui sépare ce qu'il dit de ce qu'il voudrait dire, dès qu'il parle de lui » (p. 19) [nos italiques].

Auteurs bel et bien francophones, auteurs qui, soulignons-le, représentent les «meilleurs éléments » des peuples colonisés, ou pour le dire avec l'image congolaise, « les meilleurs évolués », Sartre hisse ici Césaire, Senghor et Damas sur un piédestal radicalement refusé par le Martiniquais qui se veut «bouche de ceux qui n'ont pas de bouche », « homme cafre, homme 
juif, homme nègre ». Ces Africains et Antillais à Paris maîtrisent donc parfaitement une «prose d'ingénieurs », mais se changent en des poètes bien téméraires et proches de la Terre quand il s'agit de chanter leurs racines et définir leur identité. On y décèle le thème de l'élection du petit bourgeois qui devient le porte-parole des prolétaires et des marginaux écrasés par la classe dominante, auto-projection que produit Sartre : la poésie qu'il introduit est produite pour et par une élite, « les happy few », alors même qu'elle veut changer le sort des opprimés, des colonisés.

Quant au décalage linguistique que signale Sartre, l'écart antillais et africain, la dérive de la norme «franco-française » deviendra la « griffe » ou la «marque déposée » d'un Damas qui tonitrue dans son célèbre poème «Hoquet», contre « le Français de France, le français du Français, le français français » que lui inculque une mère adoptive, mulâtresse embourgeoisée qui oublie sa couleur, qui lui cloue le bec et lui interdit de jouer avec « la négraille ». Bref, ces auteurs en ont marre d'être appelés « nègres » et d'avoir maîtrisé la grammaire et le vocabulaire français pour mieux se soumettre et donc se taire face aux Blancs, pour subir encore, à l'heure où ils écrivent, les regards obliques et les mots d'injure par où commence cette douloureuse prise de conscience, mieux, cette crise existentielle décrite par tant de Noirs - par exemple, dans l'autofiction de Bernard Dadié, Un Nègre à Paris et dans Peau noire, masques blancs de Fanon, on peut lire : «Tiens, un nègre », «sale nègre » et «Maman, regarde le Nègre, j'ai peur » (p. 90).

Pour Sartre, les Antillais, les Africains formés dans des écoles coloniales françaises doivent opérer une radicale renaissance, et il appuie cette mutation par un syntagme répétitif à valence impérative : «Il s'agit de » est une tournure qui revient à plusieurs reprises :

«Il s'agit donc pour le noir de mourir à la culture blanche pour renaître à l'âme noire » (p. 23).

Si cette image a des résonances bibliques, et qu'elle ne laisse aucun «tiers-espace », aucun entre-deux (ou double bind) à des poètes qu'il appelle par ailleurs des évangélistes ${ }^{13}$, l'âme noire, telle que la définit Sartre, va de plus en plus puiser dans le vieux mythe du nègre. Lorsqu'il aborde quelques-uns des poèmes, Sartre sombre dans un essentialisme associant la

13. À la charnière entre le régime colonial et l'ère des indépendances, cette poésie serait «évangélique » au sens où elle annoncerait des lendemains meilleurs pour l'exploité et l'opprimé noir. Bien que Sartre ait défendu la résistance opiniâtre qui s'exprime dans ces premiers poèmes «nègres » et «malgaches », l'appellation «évangélistes noirs » a déplu à la postériorité africaine, ne fût-ce que pour la résonance trop chrétienne du mot. L'on sait combien le christianisme et le colonialisme ont fait bon ménage aux tropiques et dans les contrées coloniales. Même si Sartre emploie le mot «évangélique » au sens de "ouvrir au monde », faire connaître au monde, rendre public, les ex-colonisés français ont jugé le mot «évangélique » comme trop antinomique de marxiste. 
négritude à une nature, voire un tempérament fougueux et tempétueux, bref, à un naturalisme teinté d'euro-centrisme. De fait, un peu plus loin, à propos de la poésie éruptive, explosive de Césaire, il n'a pas peur de recourir à une comparaison on ne peut plus virile :

« Il ne s'agit pas de rejoindre la calme unité des contraires, mais de faire bander, comme un sexe, l'un des contraires du couple "noir-blanc" dans son opposition à l'autre » (p. 27).

D’autres images de ce genre, censées caractériser la poésie de la négritude, seraient insensées aujourd'hui :

«L'être sort du Néant comme une verge qui se dresse » (p. 33).

Sartre abusera encore de ces effets rhétoriques choquants en appelant la négritude un «panthéisme sexuel» (p. 33) et « une androgynie » (p. 33), par la fusion des principes mâle et femelle.

Troisième trait du style sartrien : le promoteur de la seule «poésie révolutionnaire » de son temps adapte un style démonstratif mais qui copie de très près les vers incandescents et truffés de métaphores de celui qui l'a indubitablement le plus marqué, Aimé Césaire. Car Césaire est allé le plus loin dans la réinvention de la langue coloniale; il fait « dégorger sa blancheur» à la langue de l'oppresseur, comme le remarque aussi Chevrier (1983 : 55) et il est celui à propos de qui Breton disait : «Et c'est un Noir qui manie la langue française comme il n'est pas aujourd'hui un Blanc pour la manier. »

Donc que fait le fin critique qui veut visiblement convaincre les lecteurs de l'anthologie de la radicalité révolutionnaire qu'opère le «super-langage » césairien dans un vers choisi ?

«Les mers pouilleuses d'îles craquant aux doigts des roses lance-flamme et mon corps intact de foudroyé $\gg{ }^{14}$.

Sartre glose comme suit :

«Voici l'apothéose des poux de la misère noire sautant parmi les cheveux de l'eau, "isles" au fil de la lumière, craquant sous les doigts de l'épouilleuse céleste, l'aurore aux doigts de rose, cette aurore de la culture grecque et méditerranéenne, arrachée par un voleur noir aux sacro-saints poèmes homériques [...]» (p. 28).

Bref, le style sartrien se fait lui-même nègre, effet d'une empathie totale, d'une reconnaissance même, car, comme le souligne Maximin dans son article «Sartre et le Tiers(-Monde)», le philosophe projette sans aucun

14. Sans référence complète, nous y reconnaissons un extrait de «Soleil serpent» de Césaire. 
doute ici son propre « complexe de laideur » dont Alain Buisine (1986) nous convainc dans son essai Laideurs de Sartre.

« J'ai changé. Je raconterai plus tard quels acides ont rongé les transparences déformantes qui m'enveloppaient, quand et comment j'ai fait l'apprentissage de la violence, découvert ma laideur qui fut pendant longtemps mon principe négatif, la chaux vive où l'enfant merveilleux s'est dissous, par quelle raison je fus mené à penser systématiquement contre moi-même au point de mesurer l'évidence d'une idée au déplaisir qu'elle me causait»(Les Mots, 1964, chap. 1, 1 \& 2).

Insistant sur l'enveloppe dermique du Noir comme masque inaltérable et qu'on ne peut enlever, Sartre-marxiste va toutefois interpréter la négritude comme un moment dialectique comme celui de la lutte prolétarienne.

Argumentation : la chaîne indivisible et une des subalternes (prolétaires, juifs, nègres)

Des convergences entre la condition nègre et celle d'autres opprimés, le prolétaire blanc et le juif, notamment, sautent aux yeux de Sartre qui part d'elles pour défendre la fin de l'oppression «nègre ».

La négritude est une poésie marxiste, la seule " poésie révolutionnaire », tant il est vrai qu'il trouve peu de poèmes aussi forts et renversants parmi la classe ouvrière française. Et Sartre de supposer son lecteur « fils de bourgeois », puisqu'il dit: «Je ne crois pas non plus que les travailleurs soient moins "doués" que nos fils de famille» (p. 12) [nos italiques]. En d'autres termes, le philosophe est tributaire des mêmes processus de différenciation sociale qu'induisent les catégories ou critères de « race », "classe », même sexe et religion! Inconsciemment, celui qui a une bonne conscience reproduit les mêmes schémas et les mêmes classifications (bourgeois $v s$ travailleurs, Noirs vs Blancs, etc.). De même, lorsque Sartre revient sur la fin d'une époque, celle des Colonies, il parle à la première personne du pluriel, à moins que ce soit un pur stratagème rhétorique (et que l'auteur se dissocie de ce «nous »?) :

«[...] nous ne pouvons plus compter sur les privilèges de notre race, de notre couleur, de nos techniques $[\ldots] »($ p. 21).

Ce qui prouve bien que lui-même croyait aussi dans la supériorité blanche, dans les avantages d'être et de naître avec une peau blanche, de la supériorité technique aussi de l'Occident face à l'Afrique.

Née de la rébellion contre le capitalisme du premier monde, contre la domination occidentale, la négritude se révolte contre le complexe d'infériorité instillé chez tous ceux qui n'ont pas la peau blanche. L'association [esclavage $=$ prolétarisation] et [oppression coloniale $=$ oppression marxiste] constitue le fil rouge dans la préface : 
«Le nègre, comme le travailleur blanc, est victime de la structure capitaliste de notre société ; cette situation lui dévoile son étroite solidarité, par-delà les nuances de sa peau, avec certaines classes d'Européens opprimés comme lui ; elle l'incite à projeter une société sans privilège où la pigmentation de la peau sera tenue pour un simple accident »(p. 13).

L'auteur de Réflexions sur la question juive (1947) souligna un an avant ce texte combien les catégories de la religion, de la race, de la classe (aucun mot, chez Sartre, quant aux différences de genre, à plus forte raison d'orientation sexuelle) sont toutes opératoires de différenciation sociale (Masolo 1998). Mais, s'il y a un rapprochement certain entre l'oppression des juifs et des prolétaires avec celle des Noirs, Sartre souligne aussi que le Noir a un handicap beaucoup plus lourd que ses confrères opprimés : il est Négativité pure ${ }^{15}$.

Épinglant le racisme et la peur de l'Autre, Sartre rêve d'une solidarité entre la minorité invisible juive et la minorité visible noire, ce qui reste plus que problématique (le rapport entre les deux reste biaisé, vexé...). Si le juif peut tricher, dissimuler, s'intégrer au point de sacrifier complètement sa «judéité », le Noir ne peut cacher sa négritude : différence essentielle, critère d'altérité ontologique qui bien sûr sera retenu par Fanon, et rappelé constamment par Glissant (« descendant de ceux qui survécurent», « migrant nu », pp. 66, 101 sq.). Si le Noir fait scandale, c'est à cause de son altérité inaltérable, inassimilable. La pigmentation de sa peau le classe tout en bas de l'échelle des marginalisés, embûche sur le chemin de l'égalité et de la justice, critère qui lui accorde tout de suite un rang inférieur dans la hiérarchie des classes sociales dans la société d'accueil qu'est la France d'il y a un demi-siècle. D'où, soutient Sartre, l'importance de prendre conscience de sa peau, de son corps, avant de pouvoir accéder à la revendication légitime de sa place et de son égalité, fraternité, liberté.

Sartre semble proposer cette «prise de conscience» comme un processus graduel, alors que tout Noir prend abruptement et péniblement conscience de sa négritude. Première rupture, reprendre le terme de Damas, premier «désastre » qui se produit à un âge très précoce, choc qui instaure en général une honte paralysante, pose un «mors » à la langue, faisant du Noir un mort en sursis tant cette négritude le marque comme un stigmate,

15. Dans sa Critique de la raison pure, Kant avait dénié l'humanité à la «race nègre », pendant que Hegel dît à propos de l'Afrique entière que c'est un continent hors de l'Histoire. La négativité poursuit Fanon qui, dans «L'expérience vécue du Noir » (chapitre cinq), critique Sartre parce qu'à son tour, il reprend malgré lui «le mythe du nègre » de l'animalité comme elle imprègne encore des représentations artistiques telle que la «Vénus noire ». Voir SHARPLEY-Whiting (1999), qui analyse les réverbérations du mythe de la négresse lascive et de la double attraction/répulsion qui émane du corps féminin noir dans Ourika, de Mme de Duras, La fille aux yeux d'or, de Balzac, et les traces d'un africanisme exotique et érotique dans les contes de Maupassant, notamment dans Boitelle, et les écrits de Loti et Zola, parmi tant d'autres. 
le rétrécissant. La réalité lui apprend très vite à voir son reflet dans le miroir et dans le regard de l'Autre, le Blanc. Le rapprochement entre les Noirs, les juifs et les prolétaires déplaît aux intellectuels africains d'aujourd'hui qui trouvent cette généralisation forcée, et remarquent avec raison que toutes les Colonies ne se ressemblent pas et que tous les Noirs ne se ressemblent pas. L'auteur et théoricien Valentin Y. Mudimbe s'engage dans un dialogue avec Sartre sur l'existentialisme et, à travers un jeu intertextuel, réplique à plusieurs fondements de la pensée sartrienne.

Toute la lecture sartrienne de cette Anthologie trahit une pensée manichéenne dont le préfacier serait dupe. En effet, Sartre interprète faussement la négritude comme une étape dialectique dans la quête d'égalité du Noir, aveugle pour l'émanation d'un nouveau racisme postcolonial. Mais surtout, il conçoit la poésie de cette faune nègre du quartier latin comme un racisme anti-blanc, ce que les fondateurs n'ont jamais voulu (Adotévi (1972) dans Négritude et négrologues, Depestre (1980) dans Bonjour et adieu à la négritude). C'est ce portrait en noir et blanc, cette absence de nuance qui procure au texte sartrien un caractère daté. À vrai dire, toute la démonstration « essentialiste » et eurocentriste serait inacceptable aujourd'hui, «politiquement incorrecte ». Émanant d'une politique identitaire essentialiste, qui ne rend service ni aux Antillais, ni aux Africains et Malgaches, ces trois dernières citations convaincront. Sur un poème d'amour de Senghor (1964), hymne à la beauté de la femme africaine, incarnant l'Afrique entière :

«Femme nue, femme obscure

Fruit mûr à la chair ferme, sombre extase de vin noir»

(Poèmes, Éthiopiques, «Femme noire », cité par Sartre, dans Orphée noir, p. 9).

Et Sartre de se lamenter :

«Et notre blancheur nous paraît un étrange vernis blême qui empêche notre peau de respirer, un maillot blanc, usé aux coudes et aux genoux, sous lequel, si nous pouvions l'ôter, on trouverait la vraie chair humaine [...]»(p. 9).

À la lecture de la négritude, déduit Sartre, on ne peut qu'avoir honte de sa blanchitude. Ce n'est pas exactement le but de Senghor qui, dans ses vers d'amour, fait découvrir à l'Européen la beauté de l'Afrique. L'inversion systématique qu'opère Sartre serait inacceptable aujourd'hui, à l'heure où le métissage et la pensée rhizomatique (Gyssels 2001b) font davantage respecter non pas la pensée dichotomique, mais les nuances. De même, plus loin :

«Et quand [le noir] fait l'amour avec une femme de sa race, l'acte sexuel lui semble la célébration du Mystère de l'être »(p. 33).

Un peu plus loin, cette même «louange» de la nature et de l'essence nègres fait dire à Sartre que : 
«Cette religion spermatique est comme une tension de l'âme équilibrant deux tendances complémentaires : le sentiment dynamique d'être un phallus qui s'érige et celui plus sourd, plus patient, plus féminin d'être une plante qui croît » (p. 33).

On n'en croit pas ses yeux ! Le stéréotype de la femme soumise, d'une féminité docile vs masculinité dominante fait bondir femmes et hommes qui ont congédié cette répartition réductrice et injuste des genres. Bref, le texte fondateur est farci de clichés, de préjugés et de pressentiments racistes et sexistes. À relire à rebours ce texte obsolète, un Sartre qui pérennise les fâcheux stéréotypes raciaux et sexuels apparaît. Tant il est vrai que Sartre n'a aucun mot pour la femme africaine, et que celle-ci est absente de l'anthologie. Comme partout sur le globe, les femmes ont suivi les hommes, ont dû arriver « en second lieu».

Dans ce troisième exemple d'emphase particulièrement moralisatrice, on dirait presque un prêche, dans la phrase suivante :

«Car la négritude n'est pas un état, elle est pur dépassement d'elle-même, elle est amour. C'est au moment où elle se renonce qu'elle se trouve; c'est au moment où elle accepte de perdre qu'elle a gagné, à l'homme de couleur et à lui seul il peut être demandé de renoncer à la fierté de sa couleur» (p. 42).

«[...] la Négritude, née du mal et grosse d'un Bien futur, et vivante comme une femme qui naît pour mourir et qui sent sa propre mort jusque dans les plus riches instants de sa vie » (p. 42).

Mais cette regrettable dichotomie induit de regrettables réductions, toutes découlant d'une pensée manichéenne. L'esprit vs le corps fait dire à Sartre que la négritude est avant tout une jubilation du corps, de la force physique (et phallique), et l'on sait depuis à quel point le Noir et les autres opprimés de couleur continuent d'être assimilés en premier lieu à des corps, actifs et passifs, lascifs et languissants.

\section{L'appropriation de Sartre à travers Fanon}

Quoique ce paratexte ait pris quelques rides (à cause d'une pensée binaire [homme vs femme, Blanc vs Noir], prônant l'essentialisme nègre, forçant la polarisation Noir $v s$ Blanc et les comparaisons avec les Antiques) ${ }^{16}$, la préface érige Sartre au rang de pionnier des études et de la praxis postcoloniales.

16. «Étrange chemin : humiliés, offensés, les noirs fouillent au plus profond d'euxmêmes pour retrouver leur orgueil, et quand ils l'ont enfin rencontré, cet orgueil se conteste lui-même : par une générosité suprême, ils l'abandonnent, comme Philoctète abandonnait à Néoptolème son arc et ses flèches » (p. 42) et « Dans les moments que les Orphées noirs embrassent le plus étroitement cette Eurydice, ils sentent qu'elle s'évanouit entre leurs bras » (p. 41). La dernière comparaison donnant le titre à l'essai : la "négritude » in fine se divulgue, nous échappe, ne peut être cernée, car être nègre n'est rien d'autre qu'être homme. 
Le premier à bien avoir voulu servir de présentateur et de voix publicitaire et promotionnelle à ces littératures de minorités, à ce qu'on appelle aujourd'hui littératures émergentes, écritures migrantes, Sartre reste celui sans qui les «nègres gréco-latins » n'auraient pas pu percer dans l'arène des lettres franco-françaises. Il permit à la faune nègre du quartier Latin de faire enfin entendre sa voix, après que des générations entières d'ancêtres avaient été bâillonnées. L'Entre-deux-guerres ne fut pas seulement l'éruption des mouvements indépendantistes (aussi dans l'Empire britannique), mais surtout la période où les Antillais et les Africains se mettent à publier, avec l'appui et le soutien des leaders, blancs et noirs.

Pour les études postcoloniales, trois spécialistes font office de "holy trinity »: Edward W. Said, Gayatri Spivak et Homi K. Bhabha. Les trois fondateurs se sont approprié Sartre à travers la pensée d'un de ses disciples, le Martiniquais Frantz Fanon, qui avait suivi les cours de Sartre à Paris, puis avait exercé la psychiatrie à Blida, dans l'Algérie pré-indépendante. Profondément imbibé de Sartre et de ce texte en particulier, Fanon publia en 1952, soit quatre ans après l'Anthologie, un essai qui allait être le manifeste des hérauts postcoloniaux, Peau noire, masques blancs. Allant plus loin que l'existentialiste et l'écrivain socialement engagé, Fanon montra que la colonisation était non seulement un système autodestructeur, minant à la fois le maître et l'esclave, un processus avilissant d'aliénation réciproque, mais que la colonisation était aussi une déshumanisation, donc un affront de nature psychologique, un traumatisme déséquilibrant dont les traces et les séquelles risquent de léser l'ex-colonisé longtemps après que la colonisation et l'esclavage aient été abolis. Pour l'Antillais, la colonisation avait de surcroît été précédée par et fondée sur l'esclavage, ce qui revenait à faire de l'opprimé un objet, une chose. Les concepts-clés de Fanon sont, tout en continuant la dialectique hégélienne du maître et de l'esclave que Sartre avait reprise, le «mimétisme » et la «violence du colonisé ».

Tous trois (Said, Bhabha, Spivak) se fondent largement sur les essais de Fanon (1921-1961), qui fut le disciple de Sartre, mais qui expliqua longuement son désaccord avec son maître dans Peau noire, masques blancs. Le concept sartrien du Regard, La Nausée, L'être et le néant, fut éminemment reconnaissable pour ce sujet ex-colonisé qui signera le livre de chevet des théoriciens de la postcolonialité. L'essai vendu, traduit et analysé dans les théories et études postcoloniales, Peau noire, masques blancs s'en prend à Sartre sur plusieurs points essentiels. Ainsi, Fanon s'indigne :

«Chez le juif», dit Jean-Paul Sartre, «il y a une sorte d'impérialisme passionné de la raison: car il ne veut pas seulement convaincre qu'il est dans le vrai, son but est de persuader ses interlocuteurs qu'il y a une valeur absolue et inconditionnée du rationalisme $[\ldots] »($ Fanon, p. 95).

Ce sera précisément sur le point de l'esclavage et de la traite négrière, comme facteurs historiques et générateurs de traumatismes et de malaises trans-générationnels, que Fanon va se montrer en désaccord avec le philosophe marxiste : 
«[...] mais je comprends ce "mécanisme psychologique". Or tout le monde le sait, il n'est pas psychologique, ce mécanisme. Il y a deux siècles, j'étais perdu pour l'humanité, esclave à jamais. Et puis des hommes sont venus, déclarant que tout cela n'avait que trop duré. Ma ténacité a fait le reste: j'étais sauvé du déluge civilisateur » (Fanon, p. 97).

«Mon sang d'esclave libéré n'était pas capable de s'affoler au nom de Villon ou de Taine. Une honte!»(Fanon, p. 98).

Fanon ne peut accepter que Sartre pense en couples binaires attribuant l'émotion au Nègre, et la raison au Blanc, comme l'avait malheureusement clamé aussi Senghor. Fanon se dit profondément blessé par les assertions sartriennes selon lesquelles les Noirs en général, mais surtout les « descendants d'Africains traités », les fils d'esclaves ne pourraient «goûter » les saveurs et les vertus de la civilisation européenne et de la culture française tant adulées par d'autres minorités, dont les juifs.

Face au colonisé introverti et invisible, rendu silencieux, subalterne, sans voix par le colonisateur, Fanon va aller beaucoup plus loin que Sartre et, à partir de son expérience professionnelle en psychiatrie à Blida, expliquer comment le colonisé est amené à recourir à la violence pour obtenir ses droits d'égalité et de citoyenneté. Or, Sartre et Fanon avaient montré comment une autre attitude émancipatrice et intégrationniste adoptée par le colonisé (aucun mot non plus pour la colonisée, chez Fanon) consistait à imiter docilement le maître, à «singer» servilement le colonisateur, afin d'être vu, entendu, respecté à son tour et espérer que cela inclinerait le colonisateur. Dans les hôpitaux algériens, Fanon constata à quel point la colonisation générait des névroses, et comment le colonisé recourait à la violence pour s'émanciper et se libérer du joug colonial.

Il n'étonne dès lors pas que la préface sartrienne, bien que contredite et critiquée par Fanon, trouve de multiples échos chez les trois principaux théoriciens des études postcoloniales, aussi polémique soit sa pénétrante préface tout entière construite autour d'un binarisme et d'un «malentendu » fondamental, à savoir que la négritude serait un racisme anti-blanc, une étape transitoire vers l'égalité entre les ethnicités.

Ainsi, Edward W. Said (1997) pour qui l'orientalisme est d'abord une question de regard sur l'Autre, une certaine vision qui extériorise l'Autre, qui réduit le non-Européen, le colonisé, à l'état de «chose », de décor, d'exotisme. L'orientalisme assujettit le sujet oriental, africain, antillais au rang de « commodité », de " chose ». La chosification contre laquelle manifeste Césaire dans son Discours sur le colonialisme (1955) trouve sa formulation juridique dans le premier article Code noir:

Article 1 : «Le Nègre est un meuble.»

La pétrification du regard blanc, la nullification du Noir sont des thèmes qui n'ont cessé de préoccuper la féministe postcoloniale indienne, Gayatri Spivak. Traductrice de la Grammatologie de Derrida, Spivak entend par le concept de «subalternité » la condition de celui et de celle qui n'a pas droit 
à la parole, à plus forte raison, à la réplique et à la contradiction. Dans son article «Can the Subaltern Speak ? », Spivak (1988) souligne que la femme et le/la colonisé(e) ont rarement eu le droit de parler pour eux-mêmes, que souvent leur parole a été confisquée, comme le remarque aussi Jean-Marc Moura (2003 : 73) en donnant l'exemple d'Orphée noir, précisément. À vrai dire, quelle meilleure illustration du concept de «subalterne » que cet incipit de l'introduction sartrienne, dans laquelle Sartre pose exactement cette même question d'un discours non longuement jugulé, d'une parole enfin désentravée, d'un langage libérateur mais forcément violent, d'une dénonciation sans fard que l'oppresseur risque d'écouter si seulement il veut céder la parole et écouter l'opprimé.

Pour l'Indien Homi Bhabha (1994), enfin, la « dislocation » et le « inbetweenness » sont des concepts de base qui rappellent maints paragraphes de L'être et le néant, et lorsque Bhabha décrit le déracinement et la détresse vécus par les migrants du «Tiers-Monde » dans les métropoles du «Premier Monde », « la nausée » sartrienne n'est pas loin. Traduisant Fanon en anglais, Bhabha lui accorde une place primordiale dans sa propre théorie d'hybridité et d'interstices. Bhabha ne sous-estime nullement la pérennité de ce que Fanon avait bien raison d'appeler un facteur surdéterminant, à savoir l'épiderme, marqueur de «différence négative »: le sujet (ex-)colonisé a beau imiter et s'éreinter à ressembler au Blanc, jamais il n'arrivera à être son pareil («almost the same, but not white», Bhabha, p. 89).

« L'âme noire est une Afrique dont le nègre est exilé au milieu des froids buildings, de la culture et de la technique blanche» (p. 16).

Tout Africain et Antillais en France souffre d'un double exil (intérieur et extérieur, comme l'avait exposé Salman Rushdie dans Imaginary Homelands en 1991). À l'exil chez soi (du fait du complexe colonial, voire, dans le cas de l'Antillais, généalogique, vu le traumatisme de l'esclavage) s'ajoute un pénible exil dans le pays adoptif. Le Noir dans la société blanche n'a pas d'alternative : soit il s'assimile à la culture dominante, s'intègre dans la société d'accueil, soit il plonge dans l'abattement et la dépression, dans cet état intermédiaire entre la vie et la mort, l'action et l'inaction.

Aux côtés et avec d'autres de son époque ${ }^{17}$, Sartre prit position contre la plus grande entreprise d'expansion territoriale et d'imposition hégémonique

17. Quoique d'autres avant lui aient plaidé coupables et aient reconnu les massacres dans les Colonies (Léon Bloy, André Gide dans son Journal d'Afrique, qui lui valut le prix Nobel en 1947). L'impérialisme européen et le colonialisme français sont dénoncés dans la préface aux Damnés de la terre, le second essai de F. FANON (1961). Sartre y mentionne les massacres coloniaux, notamment à Sétif, le bain de sang dans le chef-lieu du département occidental algérien, à Bizerte et ailleurs : "Rien n'arrive à Bizerte, à Élisabethville, dans le bled algérien que la terre entière n'en soit informée »(Damnés, p. 42) "Après Bizerte, après les lynchages de septembre, qui donc est descendu dans la rue pour dire assez? » (p. 59). Hanoï (l'Indochine) et Madagascar sont deux autres sites greffés dans l'esprit sartrien à cause des bains de sang (Damnés, p. 40). 
de sa culture par une politique assimilatrice et aliénante de son pays, l'Empire colonial français, avec deux points d'ancrage, les Antilles et l'Algérie. Enfin, à travers l'appropriation par Fanon, qui conteste Sartre tout au long de Peau noire, masques blancs, des intellectuels d'ex-Colonies - du Maghreb : juif tunisien (Albert Memmi, dont Sartre préfaça le fameux Portrait du colonisé, précédé du Portrait du colonisateur); des États-Unis : Africains-Américains (Gates ${ }^{18}$, Appiah, Jan Mohamed) ; dans le sous-continent asiatique : Indiens (Bhabha, Spivak) et Africains (Mudimbe) - en sont venus aux mots avec leurs anciens oppresseurs. À travers Fanon, Sartre leur donna l'argument et l'exemple pour dénoncer les «mises au pas » des populations entières sous prétexte de civilisation et d'évangélisation, de développement, et cela au prix de lavages de cerveaux, de complexe d'infériorité, de massacres et de guerres indépendantistes et néo-coloniales. Le mérite du paratexte sartrien est d'avoir défendu une parole jusque-là jugulée, d'avoir œuvré pour un changement radical des mentalités, au risque d'un certain monolithisme et d'une représentation eurocentriste.

\section{«Une date dans l'intellectualisation de l'exister noir »(Fanon)}

Dans ce numéro spécial consacré à l'esclavage, il convient de rappeler comment Sartre sous-estima les effets trans-générationnels de l'esclavage sur les «Africains déportés » (p. 13), sur les « descendants d'esclaves négroafricains » (p. 25) présentés dans l'Anthologie de Senghor. Bien qu'impressionné par Césaire et Damas, Sartre égalise la condition prolétarienne et coloniale, alors que la honte et le traumatisme, la douleur et la rage d'avoir été vendus et dépossédés, exploités et massacrés, ligotés et calcinés, giclent de plusieurs poèmes. Citons-en seulement deux. Dans «La complainte du nègre », Damas nous rappelle, à côté du complexe de bâtardise et de l'infériorité raciale, les exactions commises sur les «meubles »:

«Coups de corde noueux de corps calcinés

de l'orteil au dos calcinés

de chair morte de tisons de fer rouge de bras

brisés sous le fouet qui se déchaîne sous le fouet »(Damas, cité par Sartre, p. 11).

Son poème «Rappel», dédicacé à René Depestre, bien que cité par Sartre, proteste contre «l'indigestion de tout morceau d'histoire de France » et contre ses blessures inguérissables:

«Il est des choses dont j'ai pu n'avoir perdu tout souvenir» (Damas, cité par Sartre, p. 10).

18. Gates (1991), dans «Critical Fanonism », rappelle la dette fanonienne à Sartre. Un des plus importants théoriciens de la littérature africaine-américaine, Gates s'est à son tour beaucoup inspiré de Foucault et des post-structuralistes pour établir sa théorie littéraire africaine-américaine (ibid. 1988). 
Et lorsque Sartre fait des Antillais une « race élue parce qu'elle a souffert » (p. 24), l'amertume et l'ironie d'un Césaire, la fatigue et la rage d'un Damas nous reviennent à l'esprit. Ainsi, que Césaire énumère tout ce que les Noirs n'ont pas inventé pour mieux faire ressortir les prétentions européennes et les arrogances des colons ayant pillé et spolié les îles et leurs populations indigènes :

«Ceux qui n'ont inventé ni la poudre ni la boussole...

ils savent en ses moindres recoins le pays de la souffrance» (Césaire, cité par Sartre, p. 34).

Ne saisissant nullement l'ironie césairienne, Sartre mythifie à la place la royauté «nègre » qui, ensemble avec «le panthéisme sexuel» et «la religion spermatique », l'éloge de «l'irrationalisme nègre », ne peuvent qu'offenser et blesser. D'où la cicatrice de Fanon, tout au long de Peau noire, masques blancs, l'amenant à conclure que «Orphée noir est une date dans l'intellectualisation de l'exister noir. Et l'erreur de Sartre a été non seulement de vouloir aller à la source de la source, mais en quelque sorte de tarir cette source » (Fanon, p. 108).

En guise de conclusion, Orphée noir de 1948 marque un tournant capital dans la prise de conscience d'auteurs et d'artistes noirs en exil à Paris, mais fait aujourd'hui figure de texte daté. Il n'étonne dès lors pas que, tout au long de cette année sartrienne, le texte n'ait pas été rappelé, bien que quelques critiques et chercheurs lui aient rendu hommage ${ }^{19}$. C'est que Sartre n'est pas postcolonial dans la mesure où les théoriciens mettent en garde contre une simple inversion des termes et qu'ils entendent sous le préfixe «post» plus qu'un simple indice temporel («après») et oppositionnel (« contre »). Ils mettent au jour les écueils et les fausses trappes d'une pensée dichotomique (eux/nous) qui risque d'insuffler vie à de nouveaux sectarismes et communautarismes.

Groupe de recherche en littératures postcoloniales, Université d'Anvers.

19. Le philosophe haïtien Eddy Soufrrant (2005) intitule son article : « Le centenaire de Sartre. Hommage d'un Haïtien de la diaspora », et Rebecca PITT (2005) choisit «Reclaiming Sartre. A Review of Birchall, Sartre against Stalinism». Tanbou (Summer) : http://www.tanbou.com/2005/ReclaimingSartre.htm 


\section{BIBLIOGRAPHIE}

Adotevi, S.

1972 Négritude et négrologues, Paris, UGE/10/18.

BÉBEL-GISLER, D.

1976 La langue créole. Force jugulée, Paris, L'Harmattan.

ВНАВНА, $\mathrm{H}$.

1994 The Location of Culture, New York-London, Routledge.

Buisine, A.

1986 Laideurs de Sartre, Lille, Presses universitaires de Lille.

CÉSAIRE, A.

1955 Discours sur le colonialisme, Paris, Présence africaine.

1983 [1939] Cahier d'un retour au pays natal, Paris, Présence africaine (Préface d'A. Breton 1943).

Chevrier, J.

1983 Littérature nègre, Paris, Armand Colin.

DADIÉ, B.

1959 Un Nègre à Paris, Paris, Présence africaine.

DAMAs, L. G.

1937 Pigments, Paris, Présence africaine (Préface de R. Desnos).

1947 Poètes d'expression française, Paris, Éditions du Seuil.

DePESTRE, R.

1980 Bonjour et adieu à la négritude, Paris, Laffont («Chemins d’Identité »).

Dubois, L.

2000 «"La République métissée". Citizenship, Colonialism, and the Borders of French History », Cultural Studies, 14 (1) : 15-34.

FANON, F.

1952 Peau noire, masques blancs, Paris, Éditions du Seuil.

1991 [1961] Les Damnés de la terre, Paris, Gallimard (« Folio »).

Gates, H. L. Jr.

1988 The Signifyin(g) Monkey: A Theory of African-American Literary Criticism, Oxford, Oxford University Press.

1991 «Critical Fanonism», Critical Inquiry, 17 (3) : 457-470.

GORDON, L. R.

2005 «Sartre et l'existentialisme noir», Sartre à l'épreuve, Cités. Philosophie, Politique, Histoire, 22 : 89-97. 
GYSSELS, K.

2001a «Négritude debout: de Antilliaanse ontvoogdingsstrijd in drie fasen », Nieuwzuid, 1 (3) : 35-41.

2001b «The World Wide Web and Rhizomatic Identity: Traité du Tout-Monde, by É. Glissant», Mots Pluriels, $18: 1-17$.

http://www.arts.uwa.edu.au/MotsPluriels/MP1801kg.html

2002 «De rampspoed van L. G. Damas : etniciteit en gender in Pigments en Névralgies », Streven, 69 (3) : 240-251.

2004 «T. Morrison et M. Condé face aux B/blancs de l'Histoire : révision et révélation autour des néo-slave narratives », in J. MisRAHI BARAK (ed.), Revisiting the Slave Narratives, Montpellier, Cahiers du Cerpanac, 2 : 487-516.

LEIRIS, M.

1955 Contacts de civilisation en Martinique et en Guadeloupe, Paris, Gallimard/ Unesco.

Masolo, D. A.

1998 «Sartre Fifty Years Later: A Review of Lewis Gordon's Fanon and the Crisis of European Man », APA Newsletters, 97 (2).

http://www.apa.udel.edu/apa/archive/newsletters/v97n2/black/sartre.asp

MAXIMIN, D.

2005 «Sartre et le Tiers(-Monde)», ms. Bibliothèque nationale, Exposition temporaire.

Memmi, A.

1957 Portrait du colonisé, précédé du Portrait du colonisateur, Paris, BuchetChastel.

MOURA, J.-M.

2003 Exotisme et lettres francophones, Paris, PUF.

Mudimbe, V. Y.

1973 Entre les eaux, Paris, Présence africaine.

1976 Le Bel immonde, Paris, Présence africaine.

1979 L'Écart, Paris, Présence africaine.

1989 Shaba deux. Les carnets de Mère Marie-Gertrude, Paris, Présence africaine.

1992 The Surreptitious Speech. Présence Africaine and the Politics of Otherness, Chicago, Chicago UP.

PITT, R.

2005 «Reclaiming Sartre. A Review of Birchall, Sartre against Stalinism », Tanbou (Summer).

http://www.tanbou.com/2005/ReclaimingSartre.htm

RUSHDIE, S.

Imaginary Homelands : Essays and Criticism 1981-1991, London, Granta Books. 
SAID, E.

L'Orientalisme : l'Orient créé par l'Occident, Paris, Éditions du Seuil.

SARTRE, J.-P.

1947 Réflexions sur la question juive, Paris, P. Morihien.

1948 Orphée noir, Préface à Anthologie de la nouvelle poésie nègre et malgache de langue française, Senghor, Paris, PUF.

2001 Colonialism and Neocolonialism. JP Sartre (Translated by Azzedine Haddour, Steve brewer and Terry McWilliams), London-New York, Routledge.

SENGHOR, L. S.

1948 Anthologie de la nouvelle poésie nègre et malgache de langue française, Paris, PUF.

1964 Poèmes, Paris, Éditions du Seuil.

Sharpley-Whiting, T. D.

1999 Black Venus Sexualized Savages, Primal Fears, and Primitive Narratives in French, Durham, N. C., Duke University Press.

SOUFFRANT, E.

2005 «Le centenaire de Sartre. Hommage d'un Haïtien de la diaspora », Tanbou, (Summer).

http://www.tanbou.com/2005/ReclaimingSartre.html

SPIVAK, G.

1988 «Can the Subaltern Speak ? », in C. Nelson \& G. Grossberg (eds.), Marxist Interpretations of Culture, Urbana, Chicago University Press. 